\title{
A Case Report: Calciphylaxis Presenting as Digital Ischemia in Patient with End Stage Kidney Disease on Peritoneal Dialysis
}

\author{
Hatoon Abbas' ${ }^{1}$, Manal Alotaibi', Ralph J. Lebron² \\ ${ }^{1}$ Department of Medicine, University of Maryland Medical Center, Baltimore, MD, USA \\ ${ }^{2}$ Department of Medicine, Mercy Medical Center, Baltimore, MD, USA \\ Email: habbas@som.umaryland.edu,malotaibi@som.umaryland.edu,rlebron@mdmercy.com
}

How to cite this paper: Abbas, H., Alotaibi, M. and Lebron, R.J. (2019) A Case Report: Calciphylaxis Presenting as Digital Ischemia in Patient with End Stage Kidney Disease on Peritoneal Dialysis. Open Journal of Nephrology, 9, 49-53.

https://doi.org/10.4236/ojneph.2019.92006

Received: March 5, 2019

Accepted: May 24, 2019

Published: May 27, 2019

Copyright () 2019 by author(s) and Scientific Research Publishing Inc. This work is licensed under the Creative Commons Attribution International License (CC BY 4.0).

http://creativecommons.org/licenses/by/4.0/

\begin{abstract}
Calciphylaxis also known as Calcific uremic arteriolopathy (CUA), is a fatal disease with high mortality that mostly affects patients with end stage kidney disease (ESKD) on dialysis. In recent years the incidence of CUA has been increasing among patients on peritoneal dialysis (PD). The exact mechanism and risk factors are not well described.
\end{abstract}

\section{Keywords}

Calcific Uremic Arteriolopathy, Calciphylaxis, ESKD, PD, HD

\section{Introduction}

Calciphylaxis is rare condition that occurs in patients with chronic or ESKD characterized by vascular calcification and vessel thrombosis leading to skin ulceration and tissue necrosis [1]. Most reports describe calciphylaxis in hemodialysis (HD) patients with fewer reported cases of patients on PD.

We present a case of calciphylaxis manifesting as digital ischemia in a patient with ESKD on PD.

\section{Case Report}

34 year-old woman with end stage kidney disease secondary to diabetic nephropathy on peritoneal dialysis presented with bilateral finger pain, swelling, and numbness for three weeks. One week before presentation she noticed dark discoloration of the fingertips.

Physical examination was significant for right middle finger periungual dark discoloration, eschar on the left ring finger distal interphalangeal joint and swel- 
ling with skin sloughing of left index, middle and ring fingers. Splinter hemorrhage was also noted on left middle finger. Bilateral lower extremities with evidence of multiple palpable hyperpigmented macules on the lateral surface. Radial and ulnar pulses were absent but positive with Doppler on the right hand and diminished with positive Doppler on the left.

Initial laboratory evaluation revealed phosphorus of $9.9 \mathrm{mg} / \mathrm{dL}$ [normal range $2.5-4.5 \mathrm{mg} / \mathrm{dl}$ ] and parathyroid hormone (PTH) level of $330 \mathrm{pg} / \mathrm{ml}$ [normal range 10 - $65 \mathrm{pg} / \mathrm{ml}$. Inflammatory markers were elevated, with erythrocyte sedimentation rate of $134 \mathrm{~mm} / \mathrm{h}$ and C-reactive protein of $4.7 \mathrm{mg}$. Rheumatologic work up was significant for positive Antinuclear Antibody (ANA) with titer of 1:320. Complement levels, Anti double stranded DNA (Anti dsDNA), antitopoisomerase 1 (Anti Scl-70) and antineutrophil cytoplasmic antibodies (ANCA) were all normal.

Upper extremity arterial duplex demonstrated right distal radial artery stenosis and waveform analysis suggestive of left radial artery occlusive disease. Upper extremity arteriogram showed right distal ulnar and radial artery disease with patent arch and left patent ulnar artery, occluded radial artery and steal syndrome. Of note, the patient had an arteriovenous fistula from being on hemodialysis in the past.

Incisional biopsy was performed on the right lower leg lesions, pathology revealed calcification of the intima, involving medium sized and smaller vessels. The dermis showed fibrosis with reactive stromal fibroblasts and surrounding adipose tissue early fat necrosis. Based on skin biopsy she was diagnosed with calciphylaxis. She was started on sodium thiosulfate, cinacalcet and sevelamer. Proper wound care was ensured to avoid complications.

\section{Discussion}

Calciphylaxis is a fatal, debilitating, and poorly understood disorder that predominantly affects patients with ESKD who are on dialysis. Our patient presented with painful skin lesions for three weeks and found to have abnormal electrolytes with high calcium and phosphorus. Calciphylaxis should be considered in patients with ESRD who present with painful skin lesions or skin gangrene and having abnormal $\mathrm{Ca}, \mathrm{P}$ and $\mathrm{PTH}$.

Early diagnosis and multi-interventional treatment in patients with Calciphylaxis can lead to improve the outcome and prevent severe consequences. Our patient was treated with sodium thiosulfate, cinacalcet and sevelamer and respond well to the treatment. Wound care team was also involved in her care.

A retrospective study that performed at a single academic center between 2008 and 2017 was revealed that multi-intervention approach can be successful in treating patients with severe calciphylaxis. The study was included 24 patients who diagnosed with calciphylaxis. They have received aggressive hemodialysis, sodium thiosulfate, appropriate wound care, pain management and discontinuation of trigger medications including warfarin. Hyperbaric oxygen, cinacalcet, 
bisphosphonates, and vitamin $\mathrm{K}$ were used in some cases. The mortality rate was high at the end of the study, but the cause of death was felt to be attributable to calciphylaxis in only 4 cases. Complete or partial resolution of lesions occurred in 17 of 24 patients [2].

Recent reports suggest rising numbers of CUA cases but it is mostly limited to case reports and case series with the precise epidemiology remains obscure. Nigwekar et al. concluded that the incidence of calciphylaxis has been increasing in recent years (from 3.7 to 5.7 per 10,000 chronic HD patients) with 2.5-fold to threefold increase in mortality among those patients compared to other chronic HD patients [3].

In 2016, a nation-wide study was conducted in the Fresenius Medical Care North America (FMCNA) dialysis units reported calciphylaxis incidence rate of 3.49 per 1000 patient-years among the patients with ESKD on chronic HD [4].

In the German Calciphylaxis Registry, $10 \%$ of the total calciphylaxis patients had ESKD treated with peritoneal dialysis and $13 \%$ of the total patients with calciphylaxis had ESKD treated with renal transplantation [5]. Despite the above mentioned, still there is little known about epidemiology of CUA in patients on PD.

A single center cohort study that included 63 patients on PD found that CUA incidence rate of 9.0 per 1000 patient-year, which is higher than previous reports of CUA incidence patients of 3.5 cases per 1000 patient-year [6] [7] [8].

Bearing in mind that this was a study on a limited number of patients and in a single center it was difficult to conclude weather PD carries higher risk for developing CUA compared to HD [7]. Furthermore, many of the studied patients were on $\mathrm{HD}$ before transitioning to $\mathrm{PD}$, this alone implies worse outcomes and higher mortality which make it more challenging to generalize this study finding [7]. Another single-center study of seven peritoneal dialysis patients who developed CUA noted that five of these patients were previously on hemodialysis and were transitioned to peritoneal dialysis for access failure or inability to tolerate hemodialysis [7]. In this case report, our patient was on HD for years before transition to $\mathrm{PD}$.

A case control study by Fine and Zacharias determined that CUA incidence is higher in PD patients compared to HD with frequency of approximately $4 \%$ of the studied PD population/year [9].

The exact mechanism of presumed increased incidence of CUA in PD patients is not well described and in one report was attributed to the use of calcium containing phosphate binders [9] [10]. Other reports indicate that PD is associated with more abnormalities in phosphorus and albumin than HD, especially once residual renal function has deteriorated in HD [7] [11] [12].

Fine et al. suggested that the constant hyperphosphatemia state in PD compared to HD patients, were significant reduction in serum phosphate occurs after each treatment, could contribute to increase frequency of CUA [9]. Besides that, Fine propose that $\mathrm{PD}$ patients are more common to have a dynamic bone disease, a condition that predispose to tissue calcification in the setting of decrease 
ability of the bone to buffer additional calcium [9].

Moreover, it was found that obesity and warfarin therapy, well known risk factors for CUA, are more common in PD patients who developed CUA than previously reported prevalence of these risk factors in HD patients who developed CUA [4] [5] [7] [8] [13]. That might be the reason behind increasing the CUA incidence among these patients and not the type of dialysis itself.

\section{Conclusions}

Calciphylaxis is a fetal disease with high morbidity and mortality. However, early diagnosis and treatment can help improving the outcome and preventing severe consequences. We emphasize Multidisciplinary care to achieve clinical improvement in this critical disease.

Calciphylaxis incidences are increasing in patients with ESKD on PD compared to HD. Although some of those patients were on HD, it still affects patients who were never on HD. The exact epidemiology as well as the mechanisms and contributing factors are unclear and need to be studied more.

\section{Conflicts of Interest}

The authors declare no conflicts of interest regarding the publication of this paper.

\section{References}

[1] Jeong, H.S. and Dominguez, A.R. (2016) Calciphylaxis: Controversies in Pathogenesis, Diagnosis and Treatment. The American Journal of Medical Sciences, 351, 217-227. https://doi.org/10.1016/j.amjms.2015.11.015

[2] Harris, C., et al. (2018) Multi-Intervention Management of Calcific Uremic Arteriolopathy in 24 Patients. Clinical Kidney Journal, 11, 704-709. https://doi.org/10.1093/ckj/sfy007

[3] Nigwekar, S.U., et al. (2014) Quantifying a Rare Disease in Administrative Data: The Example of Calciphylaxis. Journal of General Internal Medicine, 29, 724-731. https://doi.org/10.1007/s11606-014-2910-1

[4] Nigwekar, S.U., et al. (2016) A Nationally Representative Study of Calcific Uremic Arteriolopathy Risk Factors. Journal of American Society of Nephrology, 27, 3421-3429. https://doi.org/10.1681/ASN.2015091065

[5] Brandenburg, V.M., et al. (2017) Calcific Uraemic Arteriolopathy (Calciphylaxis): Data from a large Nationwide Registry. Nephrol Dial Transplant, 32, 126-132.

[6] Brandenburg, V.M., Cozzolino, M. and Mazzaferro, S. (2014) Calcific Uremic Arteriolopathy: A Call for Action. Seminars in Nephrologyl, 34, 641-647. https://doi.org/10.1016/j.semnephrol.2014.09.007

[7] Zhang, Y., et al. (2016) Calciphylaxis in Peritoneal Dialysis Patients: A Single Center Cohort Study. International Journal of Nephrology and Renovascular Disease, 9 , 235-241. https://doi.org/10.2147/IJNRD.S115701

[8] Galloway, P.A., et al. (2015) Vitamin K Antagonists Predispose to Calciphylaxis in Patients with End-Stage Renal Disease. Nephron, 129, 197-201.

https://doi.org/10.1159/000371449 
[9] Fine, A. and Zacharias, J. (2002) Calciphylaxis Is Usually Non-Ulcerating: Risk Factors, Outcome and Therapy. Kidney International, 61, 2210-2217. https://doi.org/10.1046/j.1523-1755.2002.00375.x

[10] New, N., et al. (2011) Calcific Uremic Arteriolopathy in Peritoneal Dialysis Populations. International Journal of Nephrology, 2011, Article ID: 982854. https://doi.org/10.4061/2011/982854

[11] Wang, A.Y., et al. (2005) Important Differentiation of Factors That Predict Outcome in Peritoneal Dialysis Patients with Different Degrees of Residual Renal Function. Nephrology Dialysis Transplantation, 20, 396-403. https://doi.org/10.1093/ndt/gfh331

[12] Cupisti, A., et al. (2013) Phosphate Control in Dialysis. International Journal of Nephrology and Renovascular Disease, 6, 193-205. https://doi.org/10.2147/IJNRD.S35632

[13] Hayashi, M., et al. (2012) A Case-Control Study of Calciphylaxis in Japanese End-Stage Renal Disease Patients. Nephrology Dialysis Transplantation, 27, 1580-1584. https://doi.org/10.1093/ndt/gfr658

\section{Abbreviations and Acronyms}

CUA: Calcific uremic arteriolopathy

ESKD: End stage kidney disease

PD: Peritoneal dialysis

HD: Hemodialysis 